\title{
Experimental Investigation of Piezoelectric Micropumps with Single, Series or Parallel Pump Chambers
}

\author{
Yanfang Guan ${ }^{\dagger}$, Mingyang Bai, Xiangxin Meng, Yansheng Liu and Fengqian Xu \\ College of Mechanical Engineering, Henan University of Technology, Zhengzhou 450001, China. \\ $\dagger$ Corresponding author
}

(Received 6 February 2020; accepted 18 May 2020)

\begin{abstract}
Three types of piezoelectric micropumps following different configurations: single, series, and parallel connection, are developed and investigated. All the micropumps are fabricated by wet etching technology and sealed by high temperature glass bonding. They share the same dimension characteristic of diffuser/nozzle microchannels. Verifying the impact of adding series or parallel connected pump chambers on single chambers, as well as verifying the performance of the flow rate, pressure and piezoelectric transducer vibration of three micropumps have been examined. Through the comparisons between three kinds of micropumps, the results show that the flow rate of the micropumps with parallel connected pump chambers have a higher flow rate than that of micropumps with single and serial connected pump chambers under the same driving conditions. In addition, both the flow rate and pressure with the serial micropump are the lowest. The pressure of the micropump with single pump chamber is larger than other kinds of micropumps at certain driving frequencies. Consequently, increasing the pump chambers cannot always increase the performance of the micropump. This coincides with the theory analysis. Finally, the vibration performance of piezoelectric transducers with three micropumps have been carried out. The parallel transducer has a higher vibration displacement than the other two kinds of micropumps. These results have great potentials for integration into labs with a chip or microfluidic driven systems.
\end{abstract}

\section{INTRODUCTION}

The microfluidic systems play an important role in the industry along with the fast-growing economy, ${ }^{1-4}$ especially in the fields of chemistry, life science, biology and aerospace..$^{5-8}$ For instance, these systems have been used to synthesize, separate and analyze cells, medicines and DNA, ${ }^{9-11}$ thus benefiting the development of new medicine and therapy.

To successfully implement the microfluidic systems in these applications, the connection between microscale and macro environments is critical. In most cases, fluids are pumped through the system, and one of the most commonly used tools is the micropump. ${ }^{12-14}$ From the 1990s, A. Olsson put forward the first micropump with a diffuser/nozzle microchannel, ${ }^{15}$ followed by numerous micropumps being developed and improved for several decades, including a drug delivery micropump, an EHD driving micropump, a thermo-pneumatic micropump and an electroosmotic pump, etc. ${ }^{16-20}$

Although the micropumps with a single pump chamber have been widely studied recently, ${ }^{21-23}$ micropumps with series and parallel connections of pump chambers have been proposed due to their favorable pumping performance. ${ }^{24,25}$ For example, $\mathrm{Li} \mathrm{Guo}^{26}$ and Azarbadegan ${ }^{27}$ fabricated a micropump with parallel connected pump chambers when the measured flow rate is $151.7 \mu 1 / \mathrm{min}$. $\mathrm{Hsu}^{28}$ and Fangsheng Huang ${ }^{29}$ have tested the performance of a micropump with a series connected pump chamber. The results demonstrated that the series and parallel micropump possessed better performance compared to that of micropumps with a single pump chamber. However, it seemed that these studies did not compare the differences of working principle between single, series and parallel connected micropumps, which are the crucial points resulting in these performance differences. In this paper, these differences are discussed, and more detailed experimental data is given between three types of micropumps with the same diffuser/nozzle microchannels.

\section{THEORY ANALYSIS}

A schematic configuration of the piezoelectric micropumps with a single chamber, series and parallel connected pump chambers are shown in Fig. 1(a), (b) and (c), respectively. The performance of the micropump is based on a unique trait of the "diffuser-nozzle" microchannels, which has been shown to have lower flow resistance for diffuser direction flow than nozzle direction flow. ${ }^{22}$ Consequently, a reciprocating piezoelectric transducer motion results in a net flow from the left to right for the three kinds of micropumps.

The pressure $P$ and flow rate $Q$ of every inlet and outlet are shown in Fig. 1. The conductivity coefficient $C$ for every flow direction have been listed in Fig. 1.

The flow rate in the nozzle direction flow is considered to be proportional to the pressure difference between the inlet and the outlet of the nozzles. ${ }^{30}$

$$
Q=C(\Delta P) .
$$




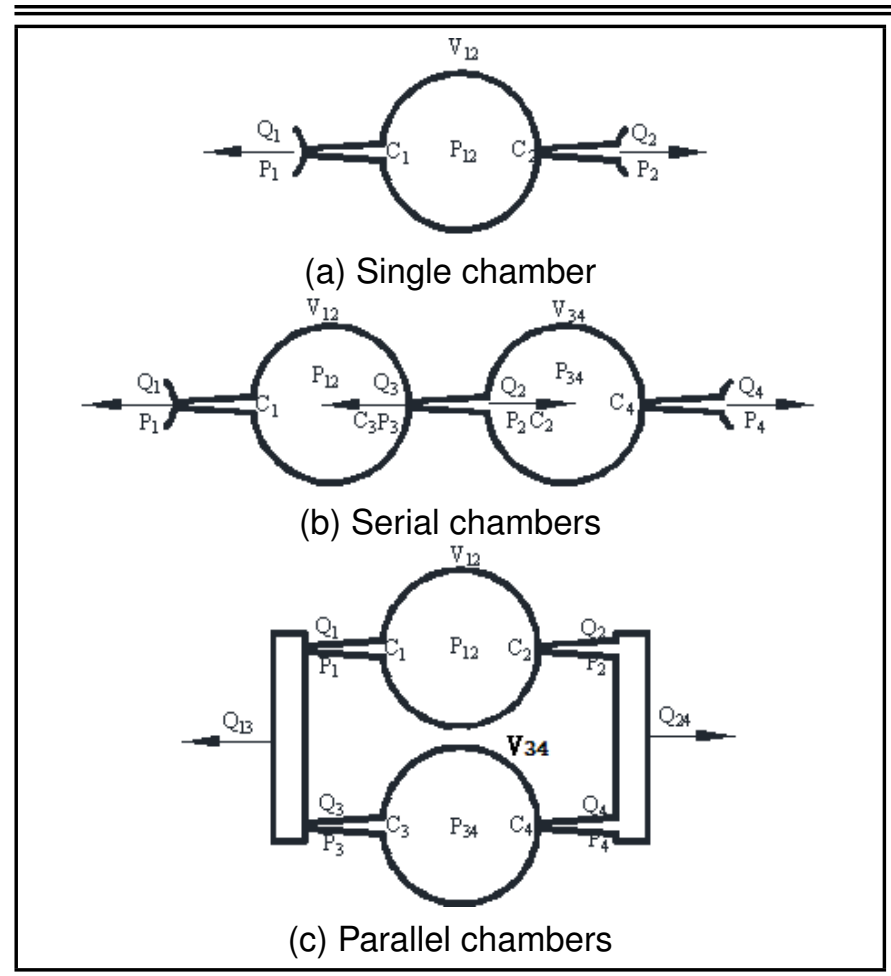

Figure 1. Schematic of micropump with (a) single, (b) series and (c) parallel pump chambers.

The analysis for the linear case is as follows.

The flow rate in the left nozzle for all three kinds of connected manners is:

$$
Q_{1}=C_{1}\left(P_{12}-P_{1}\right)
$$

while the flow rate in the right one is:

$$
Q_{2}=C_{2}\left(P_{12}-P_{2}\right)
$$

Since the fluid is considered to be impressible, therefore the sum of the flow rate should be equal to the change rate of the volume of the pump chamber, $V_{12}$, namely:

$$
Q_{1}+Q_{2}=V_{12}
$$

The change rate of volume of the pump chamber volume is usually assumed to be equal: ${ }^{22}$

$$
V_{12}=v_{0} w \sin \left(\frac{2 \pi t}{\tau}+\phi\right) ;
$$

where is the cycle time, $w=2 \pi / \tau$ is the angular frequency, and $\phi$ is a phase shift, in this paper the phase shift is assumed to be equal to $0(\phi=0)$ for every pump chamber, then $V_{34}=V_{12}$. $v_{0}$ is the volume amplitude.

Solution of Eqs. (2)-(4) for $Q_{1}, Q_{2}$, and $P_{12}$ :

$$
\begin{gathered}
Q_{1}=C_{1} \frac{C_{2}\left(P_{2}-P_{1}\right)+V_{12}}{C_{2}+C_{1}} ; \\
Q_{2}=C_{2} \frac{C_{1}\left(P_{1}-P_{2}\right)+V_{12}}{C_{2}+C_{1}} ; \\
P_{12}=\frac{C_{2} P_{2}+C_{1} P_{1}+V_{12}}{C_{2}+C_{1}}
\end{gathered}
$$

According to the same computing method for $Q_{3}, Q_{4}$, and $P_{34}$ :

$$
\begin{gathered}
Q_{3}=C_{3} \frac{C_{4}\left(P_{4}-P_{3}\right)+V_{34}}{C_{4}+C_{3}} ; \\
Q_{4}=C_{4} \frac{C_{3}\left(P_{3}-P_{4}\right)+V_{34}}{C_{4}+C_{3}} ; \\
P_{34}=\frac{C_{4} P_{4}+C_{3} P_{3}+V_{34}}{C_{4}+C_{3}} .
\end{gathered}
$$

The flow rate $Q_{2}$ and pressure $P_{12}$ for single pump chamber, $Q_{4}$ and $P_{34}$ for series connected pump chamber, $Q_{24}$ and $P_{12}+P_{34}$ for parallel connected pump chamber will be measured during experiments. Since the diffuser/nozzle are kept identical for three micropumps, so $C_{4}=C_{2}$ holds for diffuser direction flow, $C_{3}=C_{1}$ holds for nozzle direction flow. Hence, at the following:

$$
\begin{gathered}
Q_{4}-Q_{2}=\frac{C_{2} C_{1}\left(P_{3}-P_{4}\right)-C_{2} C_{1}\left(P_{1} P_{2}\right)}{C_{2}+C_{1}} \\
Q_{24}=Q_{2}+Q_{4}
\end{gathered}
$$

In addition, because of the simultaneous influence of the diffuser and nozzle direction flow on the central microchannel within the micropump with the series connected pump chambers, and the fact that at zero pressure head $P_{3}-P_{4}<P_{1}-P_{2}$ both in "supply" and "pump" mode.

So:

$$
Q_{4}<Q_{2}<Q_{24}
$$

That means the highest flow rate comes from the parallel micropump, followed by a single micropump, whereas the flow rate of the series micropump is the lowest.

The pressures between single and series micropumps have been compared, in "supply" mode when the piezoelectric transducer vibrates forward upside, leading to a vacuumed pump chamber. As a result, $P_{3}<P_{1}$, thus $P_{12}>P_{34}$. On the contrary, for "pump" mode, the result is $P_{12}<P_{34}$. Hence in the whole working process, resulted in:

$$
P_{12}<P_{34}<P_{12}+P_{34} \quad \text { or } \quad P_{12}>P_{34}<P_{12}+P_{34}
$$

where, $P_{12}, P_{34}$, and $P_{12}+P_{34}$ represent the measured pressure for single, series and parallel micropumps, respectively.

\section{DESIGN AND FABRICATION}

Three types of micropumps have been designed to investigate their pumping performance with varying operation conditions. Figure 2(a), (b) and (c) show the schematic diagram of the micropumps with single, series and parallel connected pump chambers, respectively. The geometry characteristics of the pump chambers (the diameters of P1 and P2 are $10 \mathrm{~mm}$ ), diffuser/nozzle microchannel (the length is $1.906 \mathrm{~mm}$ ) and the inlet/outlet chambers (the diameter is $4 \mathrm{~mm}$ ) are kept identical for all micropumps. ${ }^{22}$ The depth is $0.3 \mathrm{~mm}$ for the microchannels and inlet/outlet holes, whereas the depth is $1.7 \mathrm{~mm}$ for the 


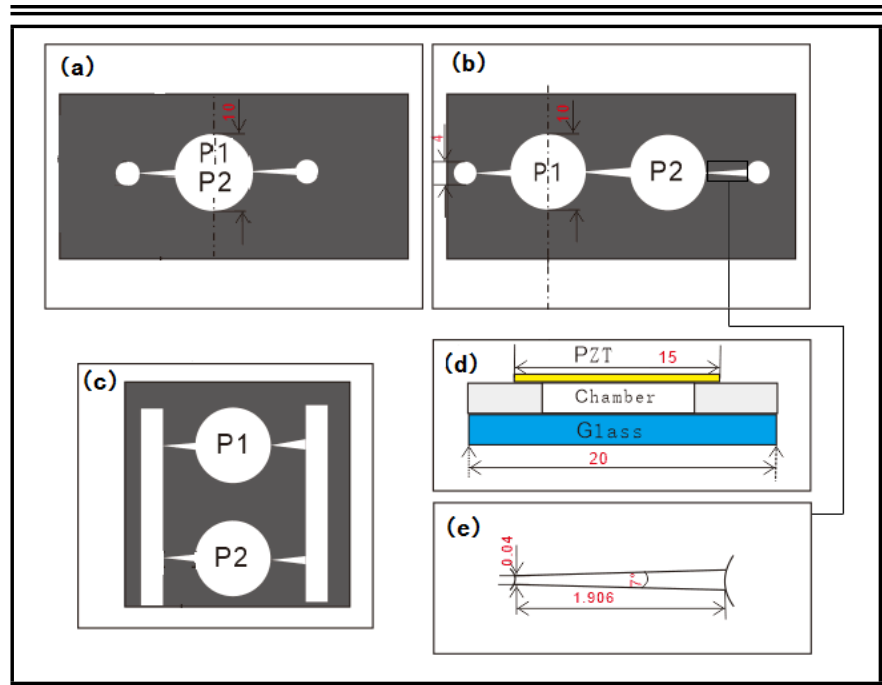

Figure 2. The structures of the micropump with single pump chamber (a), serial pump chamber (b), parallel pump chamber (c). The schemes showing the dimensions of the micropumps (d) and the diffuser/nozzle microchannel (e) (unit: $\mathrm{mm}$ ).

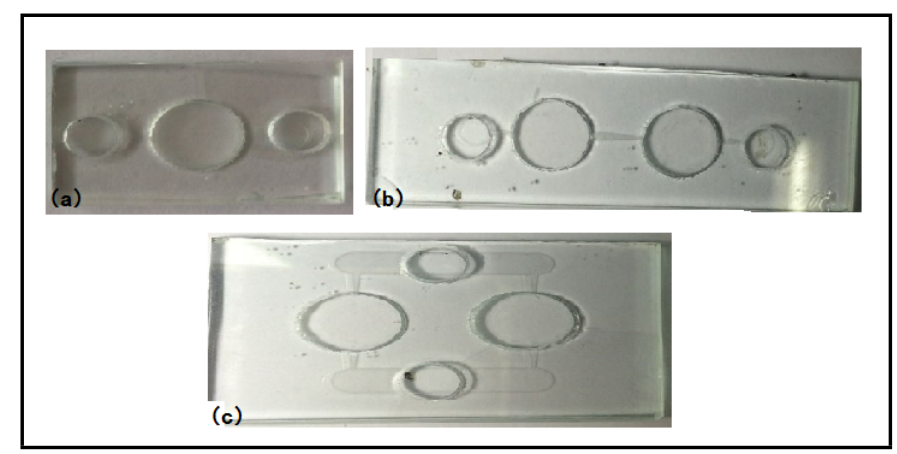

Figure 3. Photos of the single (a), series (b) and parallel (c) pump body after being wet etched on the glass wafer.

pump chambers (P1 and P2) as shown in Fig. 2(d) and (e). The pump body includes a PZT (Piezoelectric Transducer) driving layer, a glass layer with a microchannel and pump chamber and a glass layer for sealing the channel and chamber as shown in Fig. 2(d). The materials of both glass layers are Pyrex 7740 glass with $1.7 \mathrm{~mm}$ thickness. Fig. 2(e) shows the detailed geometry of the diffuser/nozzle microchannel.

To fabricate the microchannel and inlet/outlet holes for the micropumps, wet etching is adopted to etch the Pyrex 7740 glass wafer ${ }^{30-32}$ with $0.3 \mathrm{~mm}$ of depth. Afterwards, the pump chambers (P1 and P2) that are through holes on the other glass layer are made using a drilling machine. For bonding these layers together, high temperature bonding method is adopted after both layers are cleaned and dried with deionized water and a blow dryer. Fig. 3(a), (b) and (c) show the photos of the three kinds of pump bodies based on aforementioned method.

Moreover, the micropumps include two layers: the upper PDMS (polydimethylsiloxane) layer with inlet/outlet pipe for sealing the device except the inlet/outlet holes; the lower PDMS layer for fixing the PZT layer (see Fig. 3(a)). The photos of the final micropumps with series and parallel connected chambers are shown in Fig. 4(b) and (c).

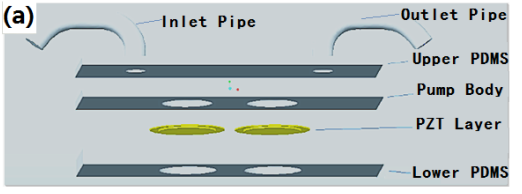

(a)

(b)
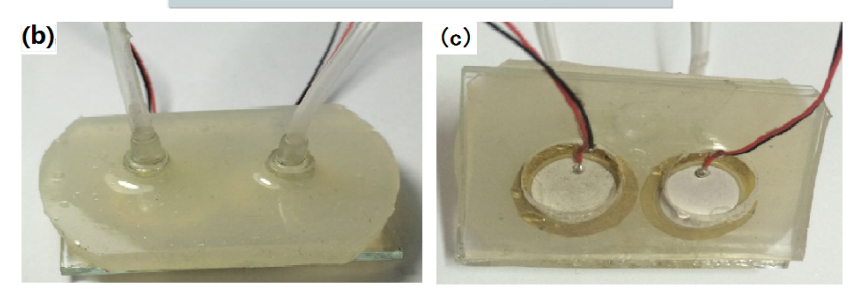

Figure 4. Schematic illustration of different layers in micropump (a) and the photos of fabricated micropumps with series (b) and parallel (c) connected chambers.

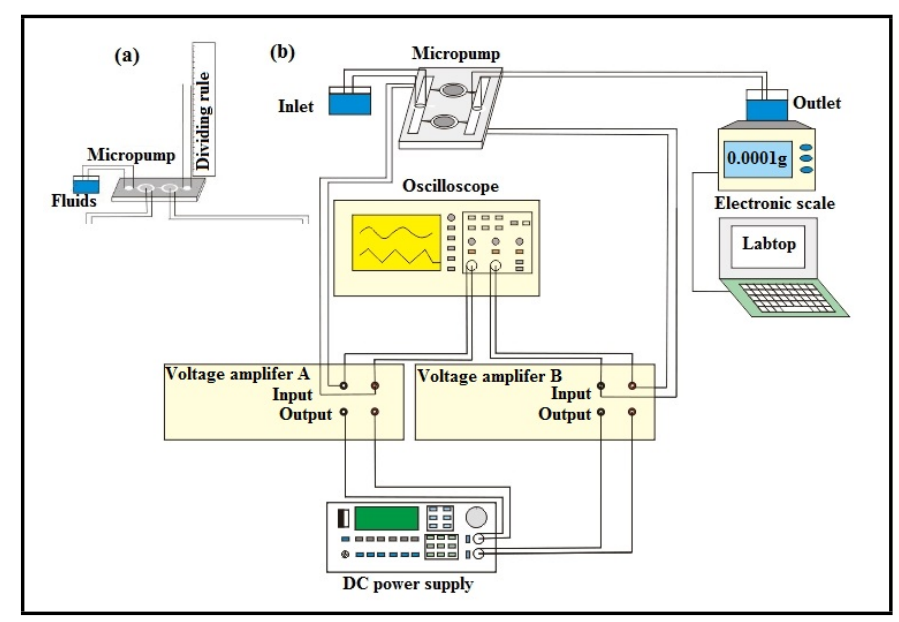

Figure 5. The schematic of the testing platform for measuring pressure head (a) and flow rate (b).

\section{EXPERIMENT}

\subsection{Micropump Performance Measurement}

The performance test of three micropumps include the flow rate and pressure measurement with sine, triangle and square driving signals combinations. The schematic of testing is shown in Fig. 5. To simultaneously actuate two PZT driving transducers P1 and P2 for the series and parallel micropumps, two set of driving and testing equipment including the signal generators, oscilloscope, analytical balances and voltage amplifiers are applied. The fluid medium is deionized water (DI water). The flow rates of the three micropumps were measured by the same procedure in Fig. 5(b). First, the inlet and outlet pipe were placed on the same plane, then the micropumps were actuated for $\Delta t=2 \mathrm{~min}$ without any difference in height between the inlet and outlet reservoirs (zero pressure head). Finally, the mass of liquid in the outlet reservoir was determined using an analytical balance, and the flow rate was calculated as:

$$
\phi=\frac{W \times 10^{6}}{\rho \Delta t}(\mu \mathrm{l} / \mathrm{min})
$$

where $\phi$ is the flow rate, $W$ is the weight of liquid in the outlet reservoir (in $\mathrm{g}$ ), and $\rho$ is the liquid density.

The pressures of three micropumps were measured by placing the outlet pipe in a vertical position and measuring the liq- 


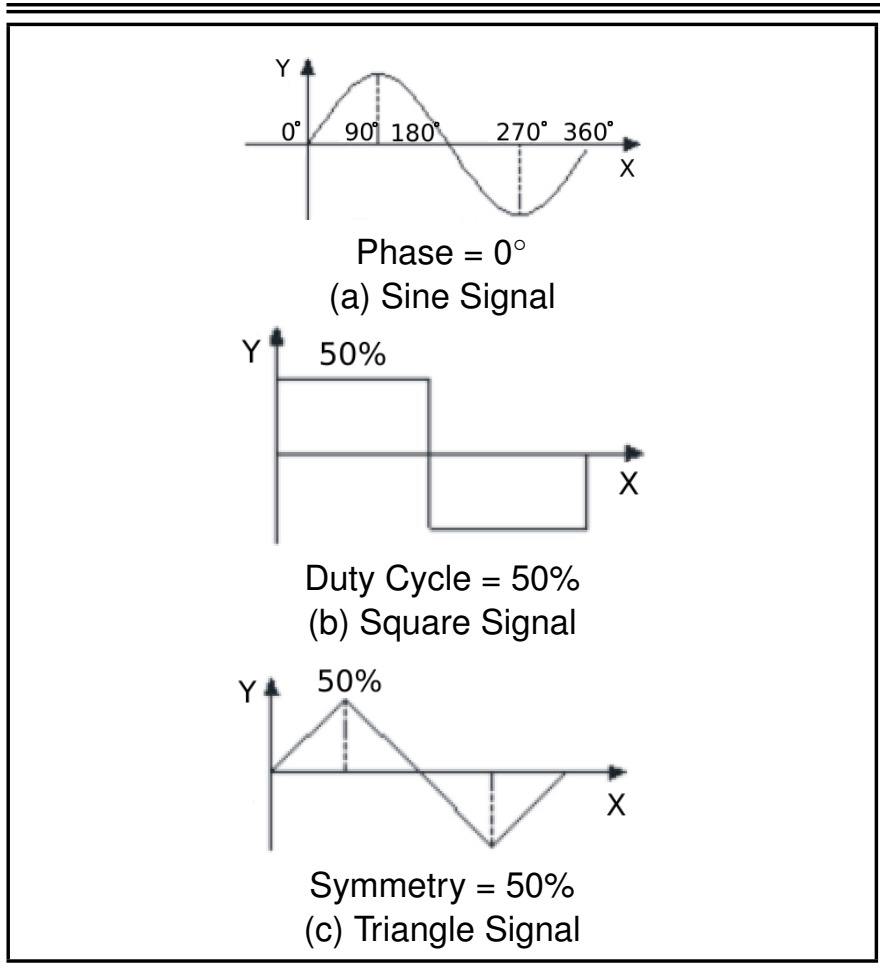

Figure 6. Phase (a), duty cycle (b), and symmetry (c) of the sine, square, and triangle driving signals.

uid height, $\Delta Z$, in Fig. 5(a), from which the pressure was calculated as:

$$
\Delta P=\Delta Z \rho g
$$

where $\Delta P$ is the pressure head at zero flow rate, and $g=$ $9.80 \mathrm{~m} / \mathrm{s}^{2}$ is the acceleration due to gravity.

The phase for sine signal driving is set to be $0^{\circ}$; the duty cycle of the square signal and symmetry of the triangle signal are 50\% as shown in Fig. 6 from DC power supply (DG1022, RIGOL, China). Moreover, the driving signals imposed on P1 and $\mathrm{P} 2$ are synchronized. The driving voltage is swept from $90 \mathrm{Vpp}$ to $130 \mathrm{Vpp}$, in $20 \mathrm{Vpp}$ steps (here, Vpp means peakto-peak value of the driving voltage). The driving frequency is swept from $0 \mathrm{~Hz}$ to $600 \mathrm{~Hz}$.

\subsection{Vibration Measurement of Piezoelectric Transducer}

The vibrational performance of the piezoelectric transducers incorporated into three micropumps is tested under forced vibration conditions with PSV400 scanning vibrometer. ${ }^{33-35}$ Fig. 7 shows the schematic of vibration displacement. The driving signals on P1 and P2 are similar to the working conditions. The material for the piezoelectric transducer is PZT-5H. The detailed parameter information is shown in table 1.

\section{RESULTS AND DISCUSSION}

The performances of three micropumps with different driving voltages, frequencies and signals are performed on P1 and $\mathrm{P} 2$. For a better visualization, the results have been interpreted in terms of three aspects: flow rate, pressure and vibration performance. All datum was conducted and processed by Matlab software.

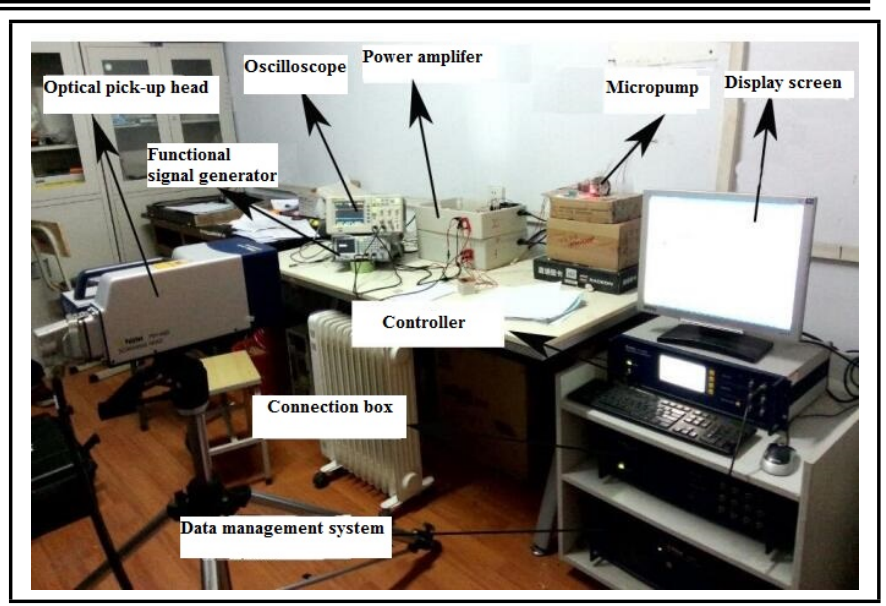

Figure 7. Experimental setup for vibrational testing.

\subsection{Flow Rate Comparisson}

The flow rates of micropump chambers are depicted in Fig. 8 as follows: single, solid and black line, series, dotted and red line and parallel dash and blue line.The driving signals imposed on P1 and P2 are a sine signal as shown in Fig. 8(a), a triangle signal as shown in Fig. 8(b) and a square signal as shown in Fig. 8(c). Three driving voltages (90 Vpp, $110 \mathrm{Vpp}$ and $130 \mathrm{Vpp}$ ) are applied as shown in Fig. 8(a), (b) and (c). The higher flow rates can be obtained through the increase of the driving voltages regardless of the type of the micropump and the input signal as shown in Fig. 8. This can be attributed to the larger displacement resulting from high driving voltage.

But when it comes to the frequency dependence, the flow rate increased at first, and then decreased along with the frequency that increased under three kinds of voltages and signals. There is a peak value (maximum flow rate value) on every flow rate curve. That is because the resonant frequency is around $50-400 \mathrm{~Hz}$, at which the volume efficiency reaches the biggest. Furthermore, the flow rate generated by a parallel micropump is higher than that of single and series micropumps under the same driving conditions. The flow rate generated by a series micropump is the least among the three signals driving. These results are in accordance with the theory analysis in Section 2. Consequently, adding parallel connected pump chambers can increase the flow rate, while adding series connected chambers reduces the flow rate.

\subsection{Pressure Comparison}

Figure 9 shows the comparison between the pressures generated by different micropumps under sine, triangle and square signals driving signals. Similarly, the driving voltages are set to be $90 \mathrm{Vpp}, 110 \mathrm{Vpp}$ and $130 \mathrm{Vpp}$. A similar change in the trend of pressures for the three types of micropumps was found for flow rates. Specifically, the pressure increases at first, and then decreases as the frequency increases. Moreover, the pressure generated by the parallel micropump is higher than that of the single in low frequency, whereas when the frequency is $200 \mathrm{~Hz}$ higher, the pressure of the single micropump is found to be larger than that of the parallel micropump, which does not agree with the trend for flow rates. Here, the reason can be the fact that vibration performance is weaker at a lower frequency, 
Table 1. Parameters of the piezoelectric transducers.

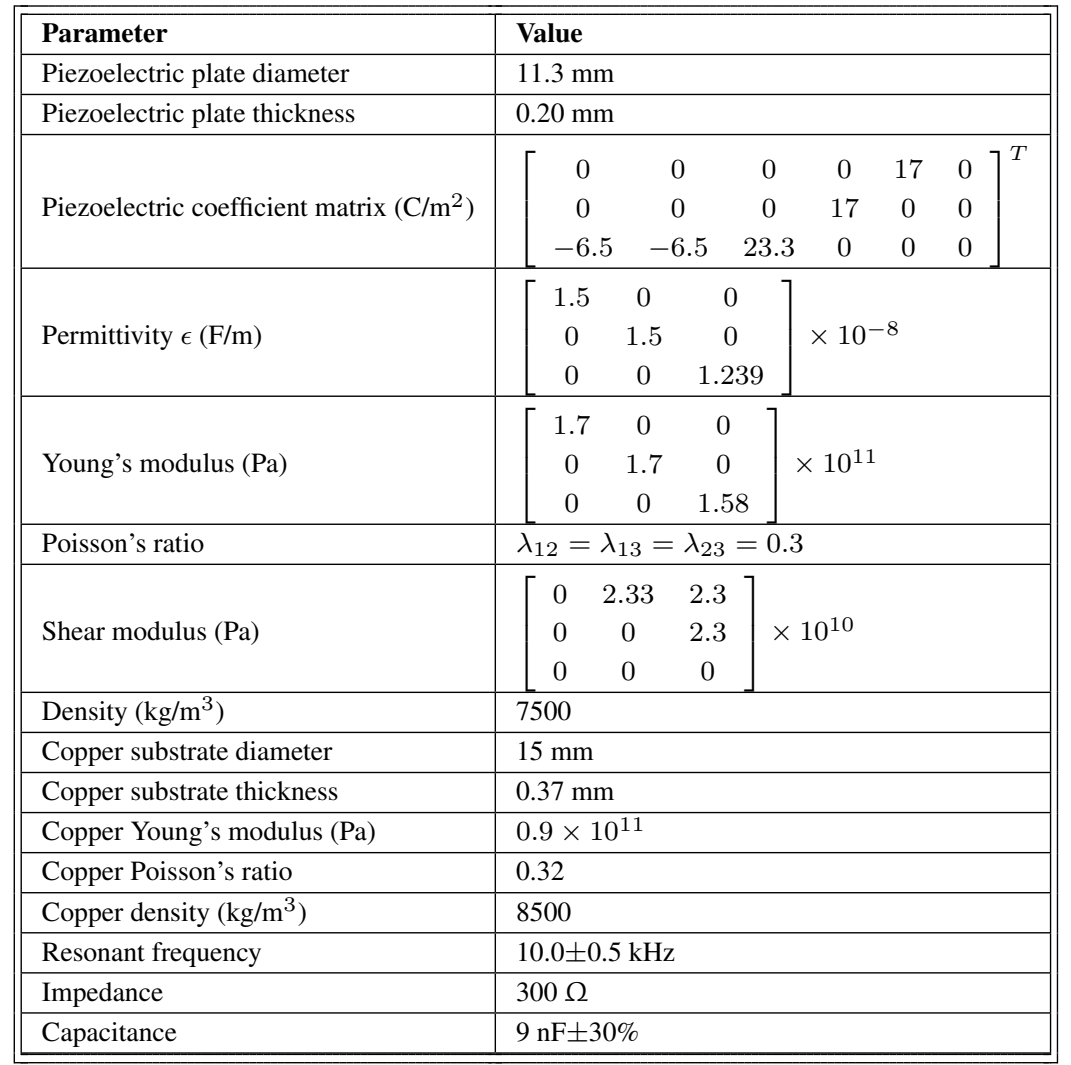

so the pressure of parallel micropump is almost twice of that of the single micropump according to Eq. (15). When the frequency increases and exceeds the resonant frequency of the PZT transducer resonant frequency, the vibration performance will be anabatic, also inlet/outlet holes for measured pressure is far away from the pump body as shown in Fig. 3(c), therefore the fluid flow becomes out of order for the parallel micropump. As a result, the pressure decreases. Note: the pressure generated by the series micropump is still the least among three micropumps.

\subsection{Vibration Deformation Comparison}

The vibrational performances are also measured for single, series and parallel micropumps. The same driving signals are imposed on $\mathrm{P} 1$ and $\mathrm{P} 2$ as sine-sine, triangle-triangle and square-square. The driving voltages are $110 \mathrm{Vpp}$ for P1 and $\mathrm{P} 2$. The phase, duty cycle and symmetry are $0^{\circ}$ and $50 \%$ for sine, square and triangle single.

As shown in Fig. 10, the vibrational displacements of the P1 and P2 piezoelectric transducers on parallel micropumps are the highest, followed by that from the single micropump lying in between P1 and P2 The PZT displacement for the series micropump is the lowest among the three micropumps. The vibration displacement of PZT responds to micropump efficiency according to the volume pump theory directly. Due to the fact that larger vibration displacement induces larger volume efficiency, this explains why the flow rate and pressure of the serial micropump are the lowest among the three kinds of micropumps. This emphasizes that increasing the capacity in the pump chamber sometimes cannot increase the microp- ump performance..$^{24,27}$ Comparing Figures 8, 9 and 10, it is seen that there are some differences regarding the resonance frequency due to the flow rate and head pressure having been measured in the outlet that is reflected in the total performance of the micropump as shown in Figs. 8 and 9. However, the displacement of every PZT transducer with three kinds of micropumps have been measured as shown in Fig. 10. The displacements reached the maximum values when the frequency was below $200 \mathrm{~Hz}$. The optimal performance with a low driving frequency is in accordance with the flow rate and pressure head.

\section{SUMMARY AND CONCLUSIONS}

In this paper the micropumps with single, series and parallel connected pump chambers have been designed and fabricated. The flow rate and pressure of three micropumps have been measured and analyzed. In order to figure out the reason for changing pressure and flow rate between three kinds of micropumps, the vibration displacement of the PZT transducer with three kinds of micropumps have been tested and discussed. The results are as follows:

(1) The change trend of the flow rate and pressure for the single, series and parallel micropumps are similar. Specifically, it increased at first, and then decreased when the frequency increased. There was a maximum flow rate and a pressure value on each curve.

(2) The flow rate and pressure generated by the parallel micropump are the largest among three micropumps (except for $200 \mathrm{~Hz}$ higher frequency) under the same driving conditions, with those of series micropump being the least. The experi- 


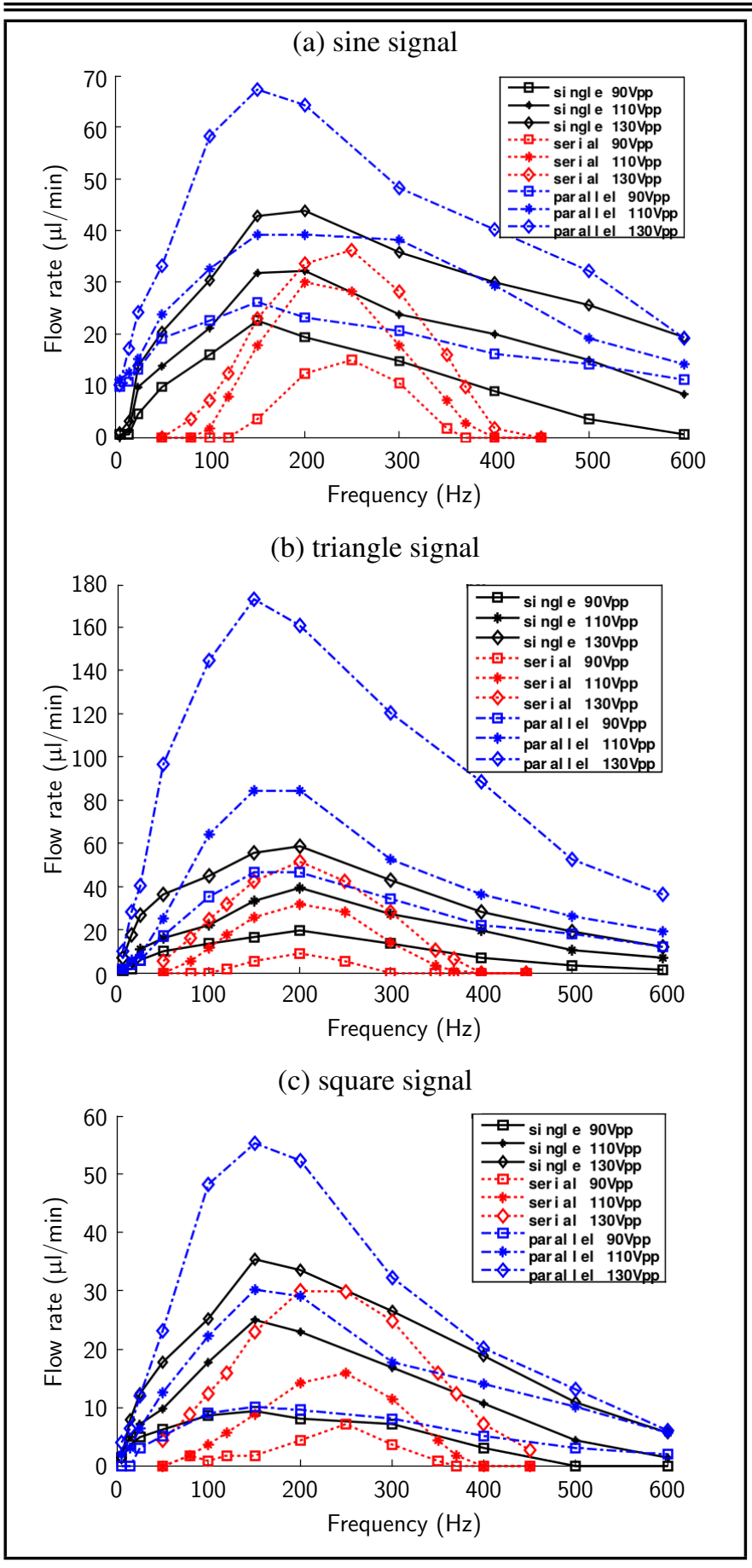

Figure 8. Flow rate of single, series and parallel micropumps with three kinds of signal driving.

mental results coincided with the theory analysis.

(3) The vibration displacement of parallel and single micropumps were larger than that of the series micropump for both P1 and P2. The displacement of the single micropump was between the $\mathrm{P} 1$ and $\mathrm{P} 2$. These results explained changing trends of the flow rate and pressure with three kinds micropumps again.

Overall, these three kinds of micropumps have their own variety of applications. For example, in the situations where the microinjected is needed in the microfluidic systems, or the use prohibits the application of electric fields in the microchannel where the fluid flow and pressure based flow are particularly desirable. (a) sine signal

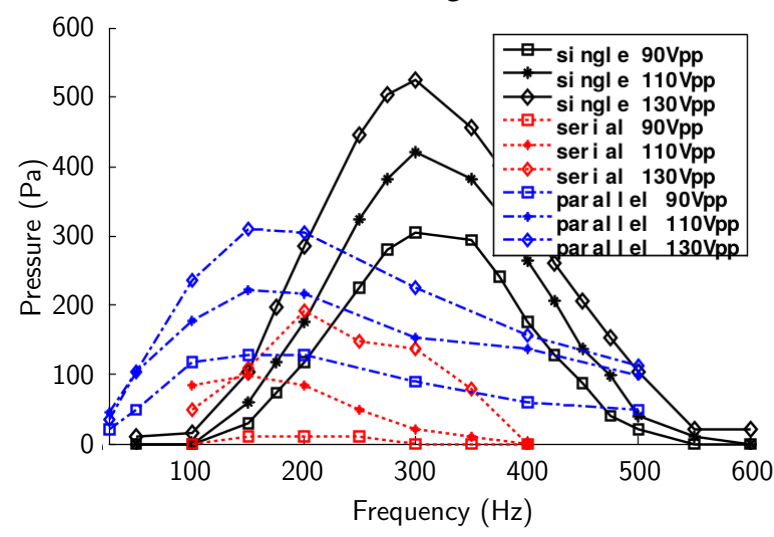

(b) triangle signal

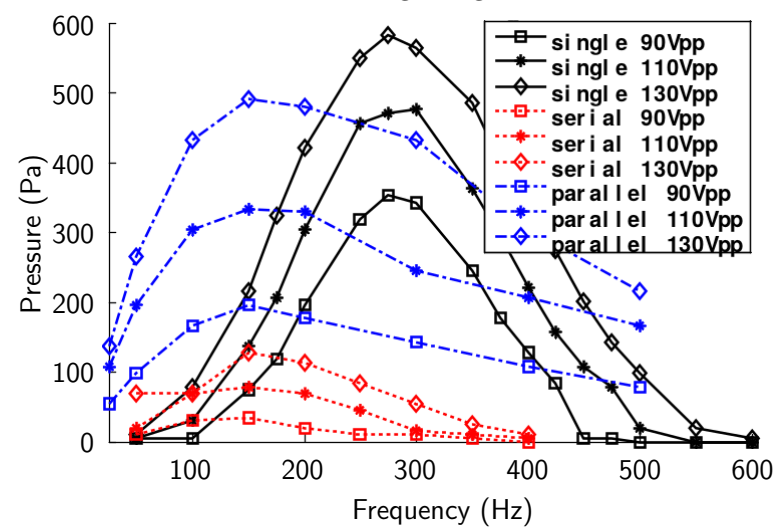

(c) square signal

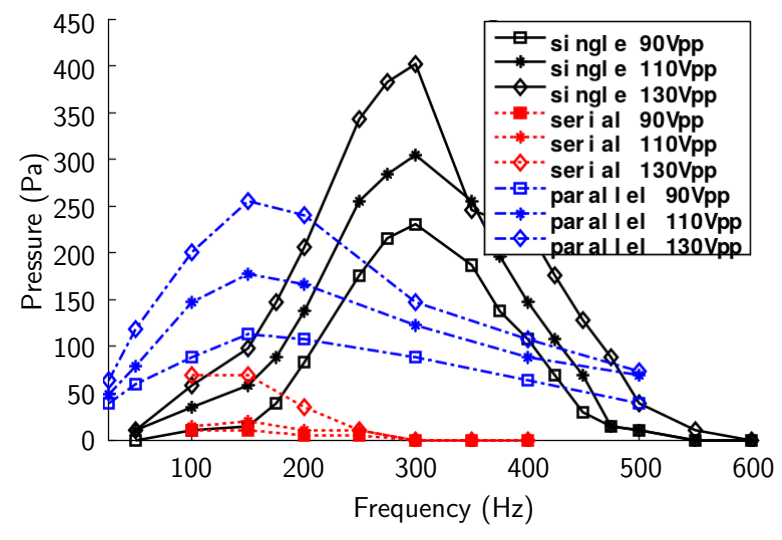

Figure 9. Pressure of single, series and parallel micropumps with three kinds of signal driving.

\section{ACKNOWLEDGEMENTS}

This project was funded by the National Natural Science Foundation of China under Contracts 51505128. It is also supported by the Young Backbone Teachers Training Program of Henan University of Technology.

The authors wish to thank Fengqian XU, graduate student, Shuaipeng Mei, Fangyu Liu, Yuhai Liu and Jian Li undergraduate students of the College of Mechanical Engineering, Henan University of Technology, for additional help during the experiment. 


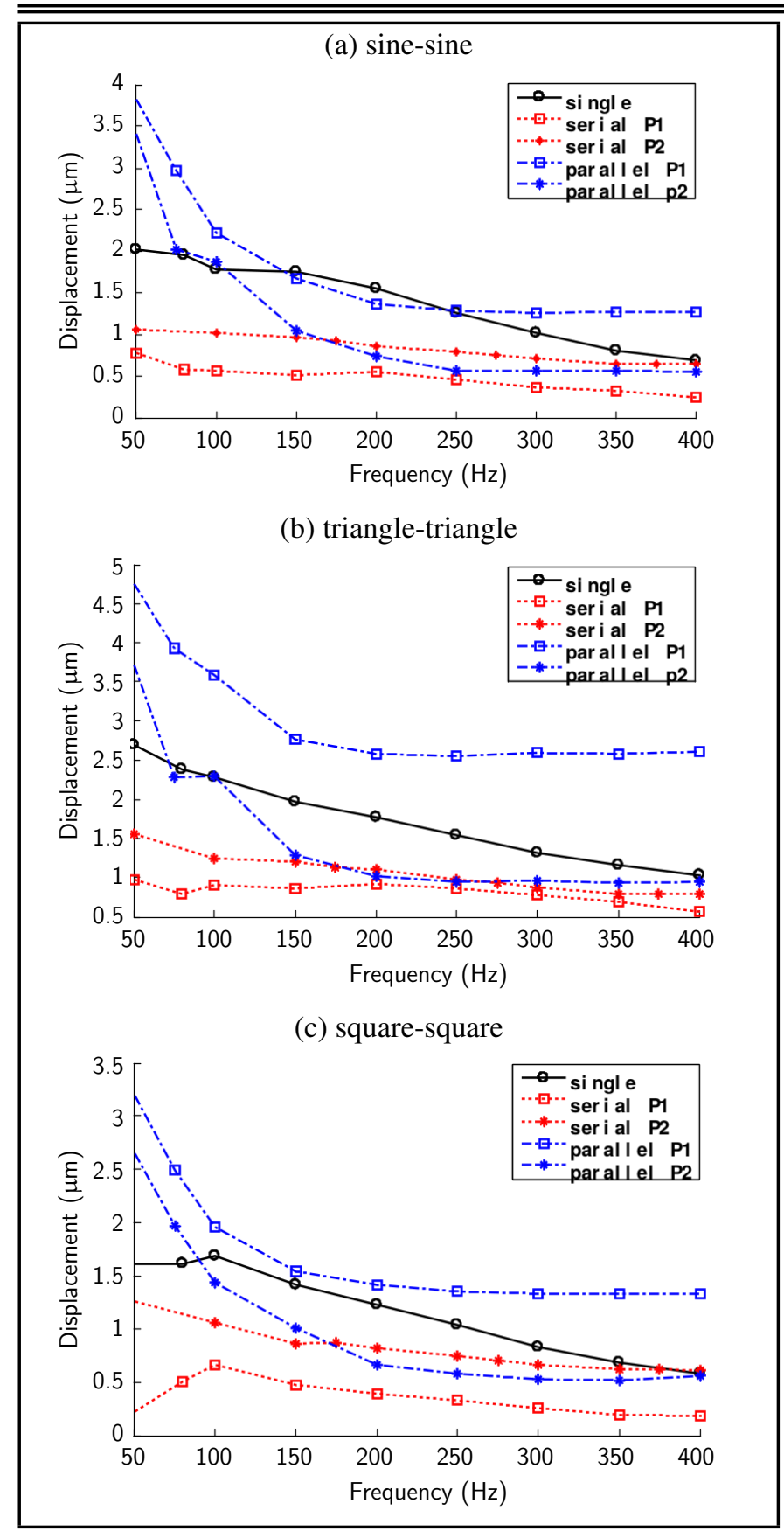

Figure 10. Vibration displacement of single, series and parallel micropumps with sine (a), triangle (b) and square (c) driving signals.

\section{REFERENCES}

1 Li, J. M., Liu, C., Zhang, K. P., Ke, X., Xu, Z., Li, C. Y., Wang, L. D., A micropump based on water potential difference in plants. Microfluid Nanofluid 11, 717-724, (2011) https://dx.doi.org/10.1007/s10404-011-0837-y

2 Tandon, V., Kang, W. S., Spencer, A. J., Kim, E. S., Pararas, E. E. L., Mckenna, M. J., Kujawa, S. G., Mescher, M. J, Fiering, J, Sewell, W. F, Borenstein, J. T., Microfabricated infuse-withdraw micropump component for an integrated inner-ear drug-delivery platform. Biomed Microdevices 12, 37, (2015) https://dx.doi.org/10.1007/s10544-014-9923-8

3 Li, R., Lv, X. F., Zhang, X. J., Saeed, O., Deng, Y. L., Microfluidics for cell-cell interactions: A re- view. Front. Chem. Sci. Eng 10(1), 90-98, (2016) https://dx.doi.org/10.1007/s11705-015-1550-2

4 Singhal, V., Garimella, S. V. and Raman, A., Microscale pumping technologies for microchannel cooling systems. Appl Mech Rev 57(3), 191-221, (2004) https://dx.doi.org/10.1115/1.1695401

5 Sugano, K., Uchida, Y., Ichihashi, O., Yamada, H., Tsuchiya, T., Tabata, O., Mixing speed-controlled gold nanoparticle synthesis with pulsed mixing microfluidic system. Microfluid Nanofluid 9, 1165-1174, (2010) https://dx.doi.org/10.1007/s10404-010-0637-9

${ }^{6}$ Ho, C. M. B., Ng, S. H., Li, K. H. H., Yoon, Y. J., 3D printed microfluidics for biological applications. Lab on a chip $\mathbf{1 5}$, 3627-3637, (2015) https://dx.doi.org/10.1039/c5lc00685f

7 Lien, K. Y., Liu, C. J., Lin, Y. C., Kuo, P. L., Lee, G. B., Extraction of genomic DNA and detection of single nucleotide polymorphism genotyping utilizing an integrated magnetic bead-based microfluidic platform. Microfluidic Nanofluidic 6, 539-555, (2009) https://dx.doi.org/10.1007/s10404-0080337-X

8 Ma, X., Jiang, S., Popescu, M. N., Uspal, W. E., Albert, M. L., Hahn, K., Kim, D. P., Sanchez, S., Reversed janus micro/nanomotors with internal chemical engine. ACS Nano 10, 8751-8759, (2016) https://dx.doi.org/10.1021/acsnano.6b04358

9 Chang, C. M., Chiou, L. F., Lin, C. C., Shieh, D. B., Lee, G. B., Three-dimensional microfluidic chip for the extraction of mitochondrial DNA. Microfluid Nanofluid 9, 489-498, (2010) https://dx.doi.org/10.1007/s10404-010-0565-8

10 Weng, X., Jiang, H., Li, D. Q., Microfluidic DNA hybridization assays. Microfluid Nanofluid (2011) https://dx.doi.org/10.1007/s10404-011-0858-6

11 Helvajian, H., Microengineering aerospace systems. Aerospace Press; American Institute of Aeronautics and Astronautics: 244-258, El Segundo, CA (1999)

12 Wits, W. W., Weitkamp, S. J., Es, J. V., Metal additive manufacturing of a high-pressure micropump. Procedia CIRP 7, 252-257, (2013) https://dx.doi.org/10.1016/j.procir.2013.05.043

13 Abhari, F., Jaafar, H., and Yunus, N. A. M., Acomprehensive study of micropump technologies. Int. J. Electrochem. Sci., 7, 9765-9780, (2012)

14 Olsson, A., Valve-less diffuser micropump. Thesis of Doctoral degree, 1-66, (1998) https://dx.doi.org/10.1016/S0924-4247(98)80059-9 
15 Ma, Y. T., Pidaparti, R. M., Simulation of drugloaded nanoparticles transport through drug delivery microchannels. Journal of nanotechnology in engineering and medicine 5, 031002-1-7, (2014) https://dx.doi.org/10.1115/1.4028732

16 Kano, I., Development of an EHD micropump to generate oscillating flow at low frequencies (effect of waveform on the EHD pumping). IEEE Transactions on industry application 48(3), 864-871, (2012) https://dx.doi.org/10.1109/TIA.2012.2191249

17 Xing, Y., Nourmohammadzadeh, M., Mendoza Elias, J. E., Chan, M. W., Chen, Z. Q., McGarrigle, J. J., Oberholzer, J., Wang, Y., A pumpless microfluidic device driven by surface tension for pancreatic islet analysis. Biomed Microdevices 18, 80, (2016) https://dx.doi.org/10.1007/s10544016-0109-4

18 Sadeghi, A., Veisi, H., Saidi, M. H., Mozafari, A. A., Electroosmotic flow of viscoelastic fluids through a slit microchannel with a step change in wall temperature. Journal of heat transfer 135/121706, 1-11, (2013) https://dx.doi.org/10.1115/1.4007414

19 Dimitry, D. F., Hassen, T, Christophe, C., Eric, C., Insulin micropump with embedded pressure sensors for failure detection and delivery of accurate monitoring. Micromachines 5, 1161-1172, (2014) https://dx.doi.org/10.3390/mi5041161

20 Hamid, N. A., Majlis, B. Y., Yunas, J., Syafeeza, A. R., Wong, Y. C., Ibrahim, M., A stack bonded thermopneumatic micropump utilizing polyimide based actuator membrane for biomedical applications. Microsyst Technol, 1-7, (2016) https://dx.doi.org/10.1007/s00542-016-2951-y

21 Hwang, S. F. and Ji, Y. M., Experimental Investigation on the Design of Nozzle/Diffuser for Micropumps. International Journal of Precision and Manufacturing, 15(4), 717723, (2014) https://dx.doi.org/10.1007/s12541-014-0392-z

22 Guan, Y. F, Liu, C. B., Structural parameter analysis and experimental study of micropumps with saw-tooth microchannel. Sensors and Actuators A: Physical, 234, 195205, (2015) https://dx.doi.org/10.1016/j.sna.2015.09.003

23 Dau, V. T., Dinh, T. X., Katsuhiko, T., Susumu, S., A cross-junction channel valveless-micropum with PZT actuatuion. Microsyst Technol 15, 1039-1044, (2009) https://dx.doi.org/10.1007/s00542-009-0878-2

24 He, X. H., Xu, L., Zhang, X. T., Yang, S., A bidirectional valveless piezoelectric micropump with three chambers applying synthetic jet. Journal of $\mathrm{Me}$ chanical Science Technology 30(9), 4015-4022, (2016) https://dx.doi.org/10.1007/s12206-016-0814-1
25 Zordan, E., Amirouche, F., Zhou, Y., Principle design and actuation of a dual chamber electromagnetic micropump with coaxial cantilever valves. Biomed Microdevices 12, 55-62, (2010) https://dx.doi.org/10.1007/s10544-0099358-9

26 Guo, L., Yan, W., Xu, Y., Valveless Piezoelectric Micropump of Parallel Double Chambers. International Journal of precision engineering and manufacturing, 13(5) 771-776, (2014) https://dx.doi.org/10.1007/s12541-012-0101-8

27 Azarbadegan, A., Cortes-Quiroz, C. A., Eames, I., Zangeneh, M., Analysis of double chamber parallel valveless micropumps. Microfluid Nanofluid, 9, 171-180, (2010) https://dx.doi.org/10.1007/s10404-009-0519-1

28 Hsu, C. J., Sheen, H. J., A microfluidic flowconverter based on a double-chamber planar micropump. Microfluid Nanofluidi 6, 669-678, (2009) https://dx.doi.org/10.1007/s10404-008-0347-8

29 Huang, F., Kan, J., and Zhang, Z., Fabrication and Performance of a Double-Chamber Serial Piezoelectric Micropump. Advanced Materials Research 655-657, 1164-1168, (2013) https://dx.doi.org/10.4028/www.scientific.net/AMR.655657.1164

30 Iliescu, C., Chen, B. T., Miao, J. M., On the wet etching of Pyrex glass. Sensors and Actuators A, 143, 154-161, (2007) https://dx.doi.org/10.1016/j.sna.2007.11.022

31 Lin, C. H., Chen, K. W., Li, T. Y., Rapid soda-lime glass etching process for producing microfluidic channels with higher aspect ratio. Microsyst Technol, 20; 1905-1911, (2014) https://dx.doi.org/10.1007/s00542-013-1980-z

32 Zhang, L., Wang, W., Ju, X. J., Xie, R., Liu, Z., Chu, L. Y., Fabrication of glass-based microfluidic devices with dry film photoresists as pattern transfer masks for wet etching. RSC Advances, 5, 5638-5646, (2014) https://dx.doi.org/10.1039/c4ra15907a

33 Ying, J., Cao, C., Jiao, Z., Loss coefficient and rectification efficiency based on valveless micropump. J. Zhejiang Univ. (Eng. Sci.) 47, 249-255, (2013) https://dx.doi.org/10.3785/j.issn.1008-973X.2013.02.009

34 Bybi, A., Grondel, S., Assaad, J., Reducing crosstalk in array structures by controlling the excitation voltage of individual elements: a feasibility study. Ultrasonics 53, 1135-1140, (2013) https://dx.doi.org/10.1016/j.ultras.2013.02.009

35 Huang, S. C. and Tsai, C. Y., Theoretical analysis of a new adjustable broadband PZT beam vibration energy harvester. Ultrasonics 105, 304-314, (2016) https://dx.doi.org/10.1016/j.ijmecsci.2015.11.027 